\title{
Estrategias de articulación entre Atención Primaria y Vigilancia en Salud y la interfaz entre los sujetos
}

\author{
Rosana Aparecida Garcia ${ }^{(a)}$ \\ Solange L'Abbate(b) \\ Jorge Arakaki(c)
}

Garcia RA, L'Abbate S, Arakaki J. Strategies for linkage between primary care and health surveillance and the interface among subjects. Interface (Botucatu). 2015; 19(54):431-42.

This paper is a qualitative study that aimed to describe the linkages between health surveillance and primary healthcare and the devices used to put these processes into effect. Data gathering was conducted through participant observation of the surveillance system, at the national level and in the municipality of Moreno, province of Buenos Aires. The data were recorded in a field diary. The results were analyzed from the perspective of institutional analysis, based on concepts developed by Lourau such as analyzer, institution and involvement. The results showed that the feelings and subjective views that circulated among the subjects involved in the process facilitated creation of devices for linking between areas and jurisdictions. The importance of this study is that the external view helped to reflect on the work processes.

Keywords: Public health. Primary health care. Health care.
Este artículo es un estudio cualitativo cuyo objetivo fue describir las articulaciones entre la Vigilancia en Salud y la Atención Primaria de la Salud (APS) y los dispositivos utilizados para que tales procesos se realicen. La colecta de datos se realizó por medio de la observación participante del sistema de Vigilancia en el ámbito nacional y del municipio de Moreno (provincia de Buenos Aires). Los datos se registraron en un diario de campo y los resultados se analizaron a partir de algunos conceptos desarrollados por Lourau, en el Análisis Institucional, como analizador, institución e implicación. Los resultados mostraron que la circulación de afectos y de subjetividades entre los sujetos involucrados en el proceso facilita la creación de dispositivos de articulación entre áreas y jurisdicciones. La importancia de este estudio consiste en una mirada externa que contribuya a reflejar los procesos de trabajo.

Palabras clave: Salud colectiva. Atención Primaria en Salud. Atención en salud. (a,b) Departamento de Saúde Coletiva, FCM, Unicamp. R. Tessália Vieira de Camargo, 126, Cidade Universitária "Zeferino Vaz". Campinas, SP Brasil. 13083-887. rosanaapgarcia@ gmail.com; slabbate@ lexxa.com.br

(c) Instituto de Salud Colectiva, Universidad Nacional de Lanús. Buenos Aires. Argentina. arakakijorge@ yahoo.com.ar 


\section{Introducción}

Este artículo presenta los resultados del trabajo desarrollado en el municipio de Moreno de la Provincia de Buenos Aires, Argentina, a partir de un intercambio entre el Departamento de Salud Colectiva (DSC) de la Universidad Estatal de Campinas - San Pablo/Brazil y la Universidad Nacional de Lanús (UNLa) de Buenos Aires a través del Programa de Centros Asociados de Posgrado Brasil/ Argentina (CAPG-BA).

El objetivo de la investigación fue analizar y comprender cómo se realizan las acciones de Vigilancia en Salud en el municipio de Moreno, cómo se articulan con los efectores de la Atención Primaria de la Salud, y qué dispositivos se han implementado para que estos procesos se hagan efectivos.

Los resultados fueron analizados en la perspectiva teórica del Análisis Institucional a partir de los conceptos analizador, de la tríada instituido/instituyente/institucionalización e implicación, desarrollados por Lourau ${ }^{1-3}$. El Análisis Institucional es un campo de conocimiento, formado a partir del psicoanálisis, las ciencias sociales y la filosofía. Según L'Abbate ${ }^{4}$ (2003), apareció en Brasil en los años 1970 a partir de algunos departamentos y grupos de investigación en las universidades brasileñas y de otras organizaciones.

Los resultados y discusiones que presentamos implican un trabajo de campo con análisis de los datos obtenidos a través de las observaciones participantes del escenario y de los procesos de la investigación registrados en un diario de campo realizado por una de las autoras.

El procesamiento y análisis de los datos se realizó, inicialmente con la reconstrucción del escenario cotidiano del Ministerio de Salud y de la Administración Nacional de Medicamentos, Alimentos y Tecnologías Médicas (ANMAT), que contribuyeron a la mejor comprensión del sistema de salud, desde la mirada de un investigador extranjero. También se realizó una breve revisión de la literatura del sistema de salud de la Argentina que ha sido objeto de innumerables trabajos científicos, razón por la cual aquí solo se hará una somera descripción con los datos más imprescindibles para comprender mejor los resultados de esta investigación, sin profundizar demasiado.

\section{El Sistema de Salud de Argentina y Vigilancia en Salud}

La Argentina es una República Federal compuesta por una Capital Federal, la Ciudad Autónoma de Buenos Aires y 23 provincias, cada una con sus propias constituciones y con autonomía para las acciones de política sanitaria. El sector salud está estructurado en tres sub-sistemas: el público, el de seguridad social y el privado. El subsistema público presenta una estructura jurisdiccional administrativa dividida en los ámbitos: municipal, provincial y nacional. Cada jurisdicción coordina sus políticas de salud a través de los respectivos Ministerios y Secretarías. El Ministerio de Salud de la Nación, desde 1990, se encarga de la normalización, regulación, planificación y evaluación de las acciones de salud que se llevan a cabo en el territorio nacional.

La Provincia de Buenos Aires se divide en 12 regiones sanitarias, las cuales tienen como misión gestionar, supervisar, monitorear y evaluar, en forma descentralizada, las políticas, planes y programas de salud dispuestos por el Nivel Central del Ministerio o planificados conjuntamente con los Municipios, hospitales provinciales y nacionales de su área geográfica.

La complejidad del sistema le imprime al área de la salud, por un lado autonomía pero por otro, una reconocida fragmentación entre los diferentes niveles jurisdiccionales, y según Belmartino ${ }^{5}$, algunos programas se articulan y otras veces se superponen, con doble regulación y fiscalización.

El Consejo Federal de Salud (COFESA), creado en 1981 con el objeto de contribuir a la integración inter-jurisdiccional y a implementar las políticas sanitarias propuestas por la Nación y las provincias, no ha logrado, a pesar de lo que dice el discurso oficial, cumplir con su propósito, en diálogos informales con actores de diferentes niveles jurisdiccionales, se ha observado que existe la percepción de que este Consejo funcionaría solo como una 'ronda de amigos'. Belmartino ${ }^{5}$ afirma, en el mismo sentido, 
que este organismo no ha alcanzado a cumplir con las expectativas del rol de coordinación asignado, tornándose confusas sus funciones reales.

El tema elegido plantea, entre otros, la relevancia de los cambios ocurridos en el contexto histórico, social y económico mundial de las últimas décadas. En este contexto, en que, por un lado se reconoce la necesidad de la protección de la salud de las personas y por otro, se abandonan las políticas de protección establecidas por los Estados benefactores, proceso cuyas características han sido objeto de discusión en diferentes países de la región y del mundo ${ }^{6-8}$. Los Estados municipales, provinciales y nacionales no pueden ser ajenos a la producción y transmisión de datos, información y conocimiento de salud, ni a la articulación y el monitoreo de los resultados de las políticas ${ }^{9,10}$.

Con este trabajo esperamos contribuir con elementos prácticos e interpretaciones teórico conceptuales al análisis de la forma en que se integran los servicios de la red de monitoreo.

\section{Metodología}

Por las características de este estudio, optamos por una conjugación de la experiencia del trabajo empírico con el análisis teórico-conceptual, lo que permite la articulación entre la teoría y la práctica. La observación participante fue utilizada como recurso metodológico para la producción de un diario de campo y la revisión bibliográfica nos permitió armar la discusión entre lo empírico y lo teórico. Los conceptos del Análisis Institucional fueron utilizados para el análisis de los materiales provenientes del campo. Es necesario aclarar que este trabajo reúne los datos provenientes de tres meses de trabajo de campo, con frecuencia semanal. Trabajar con temas relacionados a las instituciones requiere un abordaje específico, que permita describir, analizar y comprender los procesos que facilitan o dificultan la articulación entre los sujetos que forman parte de ella. Por lo tanto, concordamos con la idea de Baremblitt" ${ }^{11}$ de dibujar una "[...] carta de navegación [...]", que reúna no solo el mapa de la historia objetiva, sino también la subjetiva, y la política, entre otras; que sólo es buena para un viaje, ya que está subordinada a las singularidades del campo y los procesos, lo que hace único a quien la realiza ( $p$. 59).

Y, conforme a la postura de García ${ }^{12}$, también nos proponemos romper con los procedimientos establecidos para la producción de conocimiento tradicional, recurriendo a una "[...] audacia temible [...]" y sin el temor de ser aprendices de cartógrafos (p. 107). Es así que el camino teórico y metodológico se fueron produciendo a lo largo del trabajo de campo, donde entró y se cruzó con y en la investigación, modificando tanto las estrategias iniciales de la recolección de datos como la postura de la investigadora.

La idea que rescatamos de la cartografía, no tiene que ver con una técnica o un abordaje metodológico en sí, sino con la descripción de los procesos de trabajo y del actuar cotidiano de cada sujeto, con el intercambio afectivo y de las subjetividades existentes. Factores para los que el registro tradicional, se encuentra limitado. En términos, sobre todo, de expresar lo experimentado en esos encuentros.

Este estudio fue realizado en el municipio de Moreno, a $37 \mathrm{~km}$ de la Capital Federal, que forma parte de la región sanitaria VII, y que tiene una población de 462.242 habitantes y está dividido en seis zonas sanitarias, según Censo 2010.

En cuanto a los autores del trabajo, es importante tener en cuenta que pertenecen a diferentes lugares de inserción: uno docente de la Universidad Nacional de Lanús, una docente de la Universidad Estatal de Campinas, Brasil, y una doctoranda, de la misma Universidad, que realizó la recolección de los datos en el trabajo de campo.

Esta circunstancia no debe interpretarse como indicativa de una posición neutral en relación al objeto de investigación, ya que todos los autores estuvieron implicados en las diferentes etapas del proceso. El debate teórico se enmarca en algunos presupuestos del análisis institucional que, según refiere L'Abbate ${ }^{13}$, comenzó a partir de los años 40 y 50 en algunos movimientos que se produjeron en la sociedad francesa. Muchos autores han estudiado el concepto de implicación, y creemos que 
es muy importante señalar que la implicación como "[...] no es una cuestión de voluntad, de decisión consciente. Ella incluye un análisis de los sistemas de los lugares, el señalamiento del lugar que ocupa, que pretende ocupar, y del que se le ha designado con los riesgos que comprende"13 (p. 231).

Lourau ${ }^{14}$ afirma que la implicación "[...] es un nudo de relaciones [...]" (p. 190) y Paulon ${ }^{15}$ refiere la necesidad de incluir en el proceso de investigación, la subjetividad del investigador como una categoría analítica. En este sentido, también presupone la no neutralidad del investigador y, por otra parte, compartimos la afirmación de Lourau de que el análisis no es más para analizar al otro que a sí mismo, todo el tiempo.

Esta posición supone otro concepto de la institución, como el reformulado por Lourau a partir de Castoriadis, que reconoce tres momentos de descomposición: instituido/ instituyente/ institucionalización. Lo instituido refiere a las cosas establecidas, las normas, los conocimientos y la práctica (universalidad), lo instituyente que negaría el tiempo anterior (particularidad) y la institucionalización que sería el momento de síntesis entre los dos primeros.

El objetivo de analizar lo no dicho y las alianzas institucionales, nos llevó a utilizar el conceptoherramienta analizador. Lourau' sostiene, basado en el concepto elaborado por Félix Guattari, que el analizador es "[...] lo que revela la estructura de la institución, se burla de ella, y la fuerza a hablar [...]" (p. 284). La implicación es, en este contexto, el objeto del análisis de las relaciones que tenemos con la institución y, sobre todo, de pertenencia con nuestra institución. Según Lourau ${ }^{2}$, cada institución tiene una "[...] cara oculta $[\ldots]$ " que los analizadores ayudan a revelar permitiendo analizar lo que no es dicho en las instituciones (p. 68).

La investigadora de campo tuvo contacto con las personas que trabajaban en el campo de la Vigilancia Epidemiológica y otras las áreas que estaban directamente relacionadas con la Vigilancia o en funciones articuladoras del sistema (Cuadro 1).

Cuadro 1. Los entrevistados y la formación profesional y cargo

\begin{tabular}{|l|l|}
\hline Formación & \multicolumn{1}{|c|}{ Cargo } \\
\hline Médico & Dirección de Prevención y Control de Enfermedades Transmisibles \\
\hline Médico & Dirección de Epidemiologia y Evaluación Sanitaria \\
\hline Médico & Coordinación General de Atención Primaria en Salud. \\
\hline Médico & Dirección de Salud Mental. \\
\hline Geógrafo & Referencia de la Sala de Situación de Salud \\
\hline Enfermera & Enfermera Administrativa de la Vigilancia Epidemiológica \\
\hline Enfermera & Coordinación del Vacunatorio de Moreno \\
\hline Enfermera & Enfermera de Epidemiología \\
\hline
\end{tabular}

La investigadora fue acompañada al lugar donde funcionan la Sub-Secretaría de Salud de Moreno, Dirección de Epidemiología y Evaluación Sanitaria, la Dirección de Prevención y Control de Enfermedades Transmisibles, la Dirección General de Programas, la Coordinación General de Atención Primaria y la Dirección de Salud Mental, y comenzó la observación participante.

Fueran registrados en un diario de campo de la investigadora, sus experiencias personales y sus reflexiones acerca los momentos de extrañeza, la falta de conocimiento previo del contexto cultural y político, los afectos movilizados y el extremo dinamismo del trabajo.

Pezzato ${ }^{16}$ quien utiliza esta estrategia metodológica en su tesis de doctorado refiere que "[...] el diario es una excelente herramienta para el análisis de la vida institucional [...]" y, por lo tanto, de las implicaciones de los sujetos (p. 91-2) y refuerza la perspectiva de Hess que sostiene que esta técnica consiste en la descripción de actos organizados en torno de la vida de una institución.

Para finalizar el proceso de recolección de datos, se programó una restitución, concepto desarrollado por Lourau ${ }^{14}$ como una actividad intrínseca de la investigación y [...] concepto social-analítico [...] 
implica que se debe, y puede, hablar de algunas cosas que, en general, se dejan a la sombra [...] estas cosas serían silenciadas comúnmente (p. 51).

La reunión de restitución se realizó con la participación de todos los actores involucrados y de otros interesados, y se utilizó la técnica de oficina. Como una posibilidad de análisis de las implicaciones de los sujetos, después de la presentación de los resultados se realizó un debate acerca de los temas: conocimiento del territorio de los centros de salud, la salud como un derecho, la articulación en el trabajo en el municipio de Moreno, los conflictos en la mirada integradora jurisdiccional y la información de la salud y su utilización para la acción.

Según Lourau ${ }^{17}$, es un dispositivo que permite que la investigación avance más allá de los límites de su redacción final, que trasciende su transformación en una mercancía cultural útil sólo para el investigador o para la academia, facilitando el proceso de análisis de la implicación de los participantes y es un momento de disputas de poder, lógicas corporativas, gobernabilidad sobre los problemas críticos poco revelados "[...] la devolutiva socio analítica como un concepto, se supone que debe ser, y puede, hablar de algunas cosas que, en general, se dejan en la sombra. Estas son las cosas habitualmente silenciadas [...]"17 (p. 51).

Este momento de restitución fue reconocido por los participantes como un momento de (re) visitar su trabajo diario y valorado por los trabajadores y gestores, como importante dispositivo de gestión. La rutina del trabajo diario y el acostumbramiento que produce, los escasos espacios de reflexión acerca de la circulación de subjetividades relacionadas al quehacer cotidiano, fueron presentados como un analizador en el momento de la restitución. Este acostumbrarse a lo cotidiano no permite que la institución se revele mostrando su cara oculta.

Por supuesto que las estrategias de producción no permitían restringir un método "a priori" sino que requerían un movimiento "ad hoc" para este propósito, lo que indica construcción de un puente entre los frentes de trabajo y estar sensible a los analizadores encontrados durante la investigación y no sentirse amenazado por el nuevo mundo que se presentaba.

Con la identificación de los analizadores, nos proponemos construir una cartografía que nos ayude a analizar los movimientos del trabajo vivo en su dinámica, y que contribuya a superar el desafío de construir una mirada colectiva sobre la realidad, a partir de las percepciones de su poder instituyente. Como dicen Merhy y Franco ${ }^{18}$ " [...] provocar que usted mire los mapas como herramientas para estudiar y buscar 'oír' el ruido que hacen, incluyendo las molestias que causan [...]" (p. 2).

\section{Resultados y discusiones}

\section{¿Qué es Vigilancia en Salud? ¿Es posible una articulación con la red de Atención Primaria de Salud?}

Muchos programas de la promoción y la protección de la salud y la prevención de enfermedades, son desarrollados en los niveles jurisdiccionales, lo que despertó el interés de los investigadores en discutir cuáles son las posibles articulaciones dirigidos a la temática de vigilancia en salud, desde una mirada municipal. Acá no habría condiciones para un debate más ampliado en la perspectiva descrita pero hay un desafío de discutir sobre la posible articulación con la red de Atención Primaria de Salud.

Históricamente el concepto de vigilancia estuvo relacionado con las enfermedades transmisibles y el saneamiento de los puertos y ciudades. Actualmente existe consenso sobre la necesidad de ampliar el campo a otros problemas sanitarios.

En algunos países, Brasil entre ellos, se trabaja con un concepto de Vigilancia en Salud, que plantea el desafío de la articulación, interna y externa, entre la Vigilancia Sanitaria, la Epidemiológica, la de la Salud del Trabajador y la del Medio Ambiente.

Según Costa ${ }^{19}$, a pesar de que hay evidencias de que desde la década de 1960 tenía acciones de control de puertos, aeropuertos y fronteras, solo a partir de los años 1970 la 'vigilancia' se institucionalizaría con la creación de la Secretaría Nacional de Vigilancia Sanitaria y de la Secretaria de Vigilancia Epidemiológica dentro del Ministerio de la Salud de Brasil. 
En la Argentina, la Vigilancia se refiere a una actitud de alerta responsable sobre el estado de salud, y se constituye como una de las principales herramientas que trabaja con la interpretación, recopilación, análisis, y difusión de datos y utiliza la epidemiología para establecer la ocurrencia, distribución y factores condicionantes del proceso salud-enfermedad.

La ampliación del campo a vigilar, del individuo en particular a las poblaciones en general, fue instalando el término Vigilancia de la Salud que implica la inclusión de vigilancia de las enfermedades no transmisibles, de la vigilancia ambiental, de la calidad de los servicios relacionados con la salud (educación, agua, saneamiento, alimentos) y otros problemas sanitarios.

La vigilancia institucionalizada tiene un antecedente en la Ley 15.465 de "Notificaciones Médicas Obligatorias" promulgada en 1960, a partir del concepto de vigilancia epidemiológica, orientada a obtener información oportuna de los diferentes niveles jurisdiccionales, proporcionando la información necesaria para tomar decisiones.

La elaboración de la información es realizada por el Sistema Nacional de Vigilancia Epidemiológica (SINAVE), que recibe las notificaciones de los servicios públicos y privados de las diferentes jurisdicciones. El esquema siguiente (Figura 1) representa el flujo instituido de la información en Vigilancia Epidemiológica.

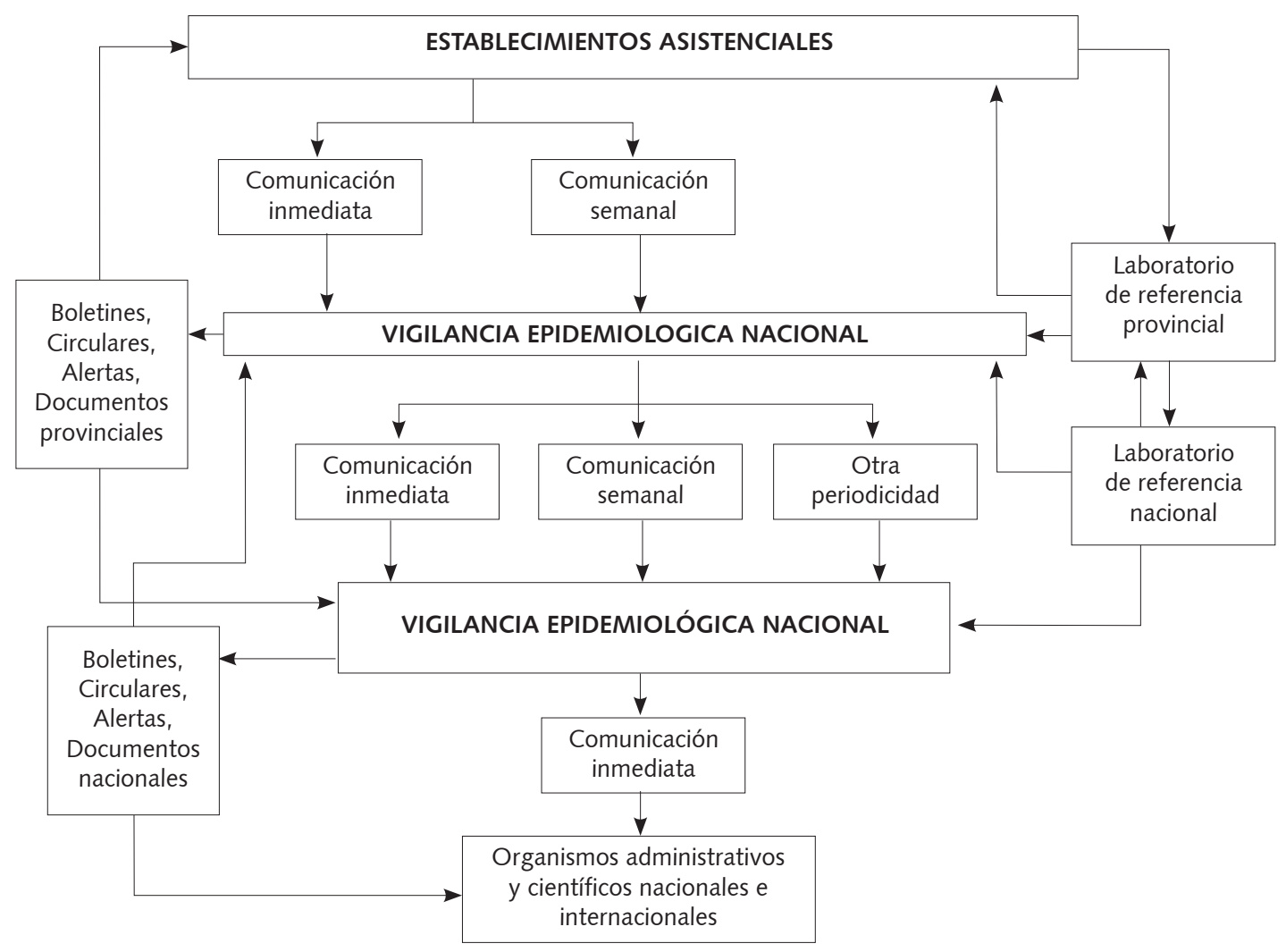

Figura 1. Flujo de la Información

Fuente: Manual de normas y procedimientos de Vigilancia y Control de Enfermedades de Notificación Obligatoria de la Nación ${ }^{20}$ (p. 12). 
El Sistema Nacional de Vigilancia en Salud (SNVS), creado en 2001, es una herramienta tecnológica de comunicación con una red de unidades ubicadas en distintos niveles jurisdiccionales y con un sitio oficial para hacer las notificaciones, con una concepción modular, lo que facilita la articulación entre las distintas estrategias de vigilancia.

La importancia de mencionar estos sistemas se debe a que nos brinda la posibilidad de analizar cómo se relacionan los sujetos que los alimentan y cómo estos flujos se producen en los procesos de trabajo del municipio. ¿Qué informaciones son producidas y qué significan para la gestión? ¿Cómo se organizan los procesos de trabajo jurisdiccionales para que los datos se conviertan en información útil para la acción y cuáles son los avances, desafíos y dificultades en estos procesos?

Un primer analizador que identificamos al observar el flujo de la información de Vigilancia Epidemiológica entre los diferentes niveles jurisdiccionales (Figura 1), es que no figura la intervención de los municipios y los datos pasarían, según el esquema del Ministerio, desde los establecimientos asistenciales a las provincias de forma directa. Interpretamos que la omisión de la jurisdicción municipal, constituye un analizador que obliga a repensar las relaciones entre los sujetos involucrados en ellas.

En el sistema de salud de Moreno, la investigadora observó que los trabajadores y gestores, pertenecientes a diversas disciplinas profesionales, aplicaban sus saberes tecnológicos específicos más allá de sus núcleos de formación, desafiando las dificultades del trabajo en equipo, y no había la preocupación por limitarse a las funciones de los organigramas tradicionales. Fue observado que había la articulación entre los profesionales, con comunicación horizontal, y asumiendo el compromiso del cuidado en salud. Los profesionales reconocen que el trabajo en equipo es un desafío, toda vez que el médico mantiene gran parte del poder instituido y el control de los procesos de trabajo en la salud.

Según los trabajadores y gestores municipales, las corporaciones profesionales, las competencias y el ejercicio profesional deberían ser repensadas, ya que no permiten que el enfermero realice muchas actividades por no estar legalmente dentro de sus atribuciones. Para las enfermeras, la capacidad de realizar una observación minuciosa y precisa es cada vez más necesaria para su práctica. Ella forma parte de su propio patrimonio científico y técnico y no representa sólo la función de describir los hechos fielmente para contribuir a la decisión del médico.

Campos $^{21}$ denomina núcleo a la demarcación de la identidad de un área de saber profesional y campo a un área de límites imprecisos hacia donde las disciplinas y profesiones se extienden. De esta forma, creemos que el campo está representado por la intersección entre las áreas, donde se crean nuevos procesos y formas de circular saberes facilitando el enfrentamiento de los problemas.

De la misma forma con el compartir los saberes y la iniciativa personal para una integración, hay cuestiones sobreentendidas, no expresadas, que necesitaban ser provocadas durante la restitución, para poder analizar los conflictos existentes y los dispositivos para superarlos.

\section{Conocimiento del territorio de los centros de salud: la salud como un derecho}

Como parte del trabajo de campo, la investigadora fue invitada a participar de una acción de supervisión de vacunación, lo que permitió conocer cuatro centros de salud y sus zonas sanitarias. Llamó la atención, encontrar en todos los centros unas placas que reproducían las palabras: "La salud es tu derecho. Defendámoslo entre todos" (grafo de la placa).

No todos los países reconocen constitucionalmente este derecho y lo aseguran. En la acción observada en territorio, podríamos asociar este derecho con algunas situaciones, con una racionalidad que parecía asegurar uno de los derechos de la población a través de una adecuada gestión de los recursos. Un ejemplo fue la distribución de vacunas, controlado por la Supervisión encargada de velar por la correcta utilización de los recursos públicos, con recuento de stock y la distribución en base a la cobertura del mes anterior.

Pero también llamó nuestra atención que una persona de la población se acercara a la supervisora de la vacunación para protestar por la falta de una vacuna responsabilizando la representante del Estado y obteniendo la orientación necesaria para dirigirse al área correspondiente, para solicitar más 
información y protestar si la situación no se resolviera a satisfacción. Nuestro entendimiento es que, en esta área en particular, de acuerdo al comportamiento observado, el llamado a defender el derecho a la salud estaba asegurado.

Siguiendo el curso del trabajo de campo, la investigadora fue informada por los profesionales de enfermería y por el conductor del vehículo sobre las condiciones socio económicas de las regiones sanitarias con tal conocimiento que mostraba con claridad cómo la construcción de un espíritu colectivo transciende las fronteras de las categorías profesionales. Esta información no fue encontrada por la investigadora en los textos de su revisión bibliográfica.

\section{Articulación en el trabajo en el municipio de Moreno}

Para facilitar el diálogo y la comunicación entre las Direcciones y los Centros de Salud, el municipio de Moreno aplicó la estrategia de establecer zonas sanitarias referenciadas por sus Centros de Salud, con sus respectivos directores, un coordinador general y una referencia de característica multidisciplinar y transversal con otras áreas, como por ejemplo la Dirección de Epidemiología y Evaluación Sanitaria, facilitando las acciones lo más tempranamente posible y una evaluación del proceso de salud en cada servicio.

A pesar de que está asumido que el núcleo de cada profesional es compartido en forma transversal, y que las diferentes implicaciones de los sujetos no se mostraron como dificultadoras de estos procesos, sin embargo, llamó nuestra atención que los afectos y subjetividades, según los sujetos no eran explorados ni analizados. El acostumbramiento al proceso de trabajo diario, decían, muchas veces no permite un momento de reflexión sobre la red de afectos producida.

El trabajo transversal de las áreas - Epidemiología, Atención Primaria de la Salud y Vigilancia fue evidenciado a través de las situaciones de observación del cotidiano laboral. Muchas veces la investigadora observó ejemplos de alguna necesidad urgente, y las respuestas no se definían desde un núcleo disciplinar o un sector específico, sino desde un campo de conocimiento compartido por todos los profesionales intervinientes.

Las herramientas para esta integración forman parte de lo que Merhy, Feuerwerker y Ceccim² llaman "[...] maletín tecnológico [...]" conformado por las tecnologías blandas, el espacio de relaciones entre sujetos; las tecnologías blanda-duras, los saberes estructurados y las tecnologías duras, los instrumentos y equipamientos (p. 151). La disposición de los trabajadores para una colaboración transversal, demuestra que hay una circulación de estas tecnologías en la perspectiva de un 'bucle', el trabajo se potencia y los sujetos se empoderan.

Algunas acciones demostraban la integración y la articulación en la Vigilancia Epidemiológica y otras áreas y sectores, como es el caso de la búsqueda activa de vacunación retrasada, que podría ser nada más que un instrumento normativo, instituido por un sector institucional aislado para el cual las normas y reglas pueden y deben ser aplicadas. Sin embargo, pudo observarse una acción compartida de los sectores correspondientes - Dirección de Epidemiología y Evaluación Sanitaria, Dirección de Prevención y Control de Enfermedades Transmisibles, Dirección General de Programas y la Coordinación General de Atención Primaria.

El Programa Nacional de Inmunización ha instituido algunos instrumentos y planillas para el área y hay una rutina de vigilancia de eventos supuestamente atribuidos a la vacunación o inmunización. A su vez, la provincia también tiene instrumentos propios para los efectos que considera convenientes, que pueden o no ser coincidentes con los de la Nación. En el municipio de Moreno hay instrumentos propios para la generación o la utilización de los datos, creados en un movimiento autónomo, instituyente que contribuye a brindar nuevos significados y sentidos para los servicios de salud.

\section{La 'mirada integradora' jurisdiccional: un momento de conflicto}

Las clásicas dicotomías entre planificar y hacer, entre pensar y ejecutar y entre la prevención y promoción y la asistencia nos parece que complementan la explicación del escenario presentado. La 
superación de las fragmentaciones institucionales es un desafío complejo y requiere también superar estas dicotomías.

Durante el debate en el momento de restitución pudimos percibir también otro analizador: existe un discurso instituido desde el nivel nacional, que afirma "[...] la Nación y las Provincias formamos un solo equipo [...] y que hay una "[...] mirada integradora $[\ldots]$ "23 (p. 6-7) y al inquirir sobre él, la impresión fue que aquel discurso flaqueaba y no se sostenía en la realidad. Analizadores como gestos, silencios y sonrisas mostraban contradicciones entre las jurisdicciones nacionales, provinciales y municipales, que no habían sido reveladas.

A partir de este discurso instituido nacional, la investigadora fomentó debates, y después de muchas entrelíneas, algunas personas manifestaron el reconocimiento de una desarticulación jurisdiccional, veladas en los espacios de gestión. Reconocieron también que había programas nacionales que se superponen con los proyectos de la provincia y el municipio.

Muchas intervenciones nacionales que no articulan con la provincia y el municipio fueron percibidas, lo que lleva a una ardua tarea por delante, la de producir una información útil, que respete las necesidades de los servicios locales de salud. Si bien hay una afirmación oficial de esta integración, hay en contrapartida, procesos de trabajo en nivel provincial y municipal, que no apuntan en esta dirección, y que la práctica cotidiana contradice.

La autonomía, con su organización política y administrativa en niveles jurisdiccionales, debería ser repensada y dirigida hacia nuevos estudios empíricos y prácticos, para facilitar el cuestionamiento de los poderes instituidos, y para poder dar respuesta a las inequidades sociales, a partir de los análisis del lugar y de las posiciones ocupadas por cada sujeto en las políticas, en el nivel macro y el micro.

Otro momento que llamó la atención en la investigación fue la expresión utilizada por un trabajador, refiriéndose a una sensación de que la mirada nacional podría ser representada como una persona en una "torre de un castillo", mirando a lo lejos, sin conocer el territorio ni las diversidades regionales.

Con esta afirmación podemos ver la necesidad de un análisis de las implicaciones de estas instituciones y sus sujetos, de las cuales Lourau ${ }^{3}$ decía que eran las responsables de hacer aparecer "[...] de un solo golpe, a la institución invisible [...]". Pero el autor advirtió también acerca de la importancia de ser cuidadoso y de no caer en la trampa de una "[...] persecución revanchista [...]", en "[...] denuncias impotentizantes [...]", o en "[...] alianzas espurias [...]" (p. 52).

\section{La información de la salud y su utilización para la acción}

El concepto y las formas de la vigilancia en salud coherentes con el desarrollo histórico de la salud pública en la lucha contra las enfermedades adoptaron prácticas, verticales y autoritarias, que hoy se encuentran con la aparición de corrientes de reorganización de los procesos de trabajo y de las herramientas utilizadas, lo que lleva a enfrentamientos dentro de la complejidad tanto de los servicios de salud como de la sociedad como un todo.

Otro momento observado por la investigadora en el campo fueron las reuniones periódicas entre las diversas áreas, para la construcción de la llamada de 'sala de situación'. La sala de situación es una de las estrategias difundidas internacionalmente por la Organización Panamericana de Salud (OPAS), con el objetivo de contribuir a planificar, definir políticas de la salud, evaluar las acciones, realizar vigilancia de la salud pública, dirigir la respuesta de los servicios de salud en situaciones de emergencia y divulgar información sobre salud en la comunidad.

La sala de situación, dentro de las regiones sanitarias, puede articular en forma dinámica y permanente el diagnóstico de las problemáticas de salud de la población, generando respuestas adecuadas para las necesidades locales. Además, pueden contribuir a un proceso de producción de información permanentemente actualizado, con devolución rápida a los servicios de salud, y transformar los datos en información para la acción, teniendo en cuenta la particularidad de los diferentes territorios ${ }^{24}$.

Este dispositivo instituido no se limita a un espacio virtual de procesamiento de datos para producir gráficos, tablas o mapas: el equipo transformó esta tecnología en trabajo vivo, siendo de acceso a 
un equipo multiprofesional y a favor del interés de cada servicio y zona sanitaria y con la potencia de transcendencia a los núcleos de conocimiento (movimientos instituyentes).

Según Merhy, Feuerwerker y Ceccim²2, hay una diferencia entre actor-autor: "[...] el actor permanece 'actuado' pero que aporta algo a la obra [...] ya el autor asume su propia invención, se torna productor de algo [...]" (p. 154). Este autor, en este mapa analítico, continúa sus cuestionamientos, ahora relacionados a la información que retorna de forma tardía, perdiendo la oportunidad y el sentido que podría tener para quienes ejecutan las acciones.

La dicotomía entre ejecutar y planificar fue señalada, mencionándose como ejemplo el de los datos de la mortalidad general. En el momento de restitución los trabajadores y gestores discutirán que la información llegaba después de un año, aproximadamente, lo que no tiene sentido si se espera una respuesta rápida del sistema. En el espacio del trabajo vivo, el equipo creó una planilla donde todos los casos de óbito son rescatados de forma temprana, permitiendo que las informaciones lleguen con mayor velocidad a los servicios de salud, sin necesidad de esperar los datos de la provincia.

Al mismo tiempo en que la información en salud constituye algo que permite planificar y gestionar en el territorio, no podríamos dejar de estar atentos a las ausencias de datos. Durante la reunión para la restitución uno de los sujetos abordó que si no transformamos los datos en algo que tenga valor y utilidad para los servicios de salud, estaremos cometiendo el riesgo de trabajar para alimentar un "cementerio de datos". Ante la sorpresa de la investigadora, expresada por esta denominación, es importante agregar que el trabajador se mostró conmovido al reflexionar durante el debate propuesto en la restitución sobre el esfuerzo vano que implica generar un dato que no va a tener valor de uso.

Durante los debates acerca de los flujos de la Vigilancia (Figura 1), surgió una pregunta: “¿cuál sería el tiempo de retorno eficaz para las provincias y municipios, para que la información fuera oportuna para la acción? y ¿habría respeto para las informaciones que los datos traen?

Finalizando las discusiones, no podría dejar de hablar sobre la circulación de afectos entre los trabajadores de la salud del municipio de Moreno. Todos, sin excepción, señalaron el encanto de trabajar con la gente, con la posición que ocupan, el orgullo por lo que producen, el empeño y la responsabilidad por disminuir los riesgos en salud, el placer en el hacer y trabajar cotidiano. El flujo de afectos y subjetividades se resiste a ser registrado, solo se siente. Se siente con el corazón, se vive con la conmoción.

\section{Consideraciones finales}

La construcción de una "carta de navegación" no es una tarea fácil y nos expone a los temores de no ser "metodológicamente correctos", a las inseguridades de las desviaciones y a las turbulencias del terreno. Con todas esas prevenciones, nos entregamos a la investigación, dispuestos a andar el campo y a ser cambiados por él.

La posibilidad de observar cómo los discursos nacionales instituidos se confrontaban en el espacio municipal en un contexto de cierto temor y reacio a hablar sobre esto, hace pensar cómo utilizar y profundizar a partir de este analizador la discusión sobre cómo construir otros procesos de gestión, con confianza y efectividad.

Hay problemas de fragmentación reconocidos por los responsables del gobierno y una defensa discursiva de supuestas articulaciones de los sistemas de información con las prácticas de los servicios. La Nación, la Provincia y los Municipios se presentan como tres niveles separados, muchas veces aislados, que responden de diferentes formas al mismo problema.

La división del municipio estudiado en zonas sanitarias y los procesos de trabajo que se desarrollan constituyen, a nuestro criterio, espacios estratégicos con potencialidad para responder con acciones efectivas y oportunas a los imprevistos que nos plantean las diferentes situaciones sanitarias. Aptas también para la discusión de nuevos procesos de trabajo y para abordar nuevos desafíos en las relaciones de poder.

Persisten muchos desafíos para nuevas construcciones: las corporaciones profesionales se comportan todavía como defensoras de leyes que muchas veces no permiten que las tecnologías 
blandas se apliquen. Los trabajadores y gestores dijeron que en el área de Vigilancia hay una gran demanda y que los enfermeros son un apoyo importante del sistema y que en las relaciones tradicionales, reproducidas en muchas instituciones, el enfermero es solo un ayudante del médico.

\section{Agradecimientos}

Al Dr Adrian Gayoso, la Dra Cristina Cipolla y al Dr Joaquin Narbeburu que permitieron la inserción de la investigadora en el campo y facilitaron las discusiones.

A los enfermeros Roberto Gabriel Pawlowicz y Luciana Franco del Hospital General de Agudos de Buenos Aires.

Al Dr. Raúl Forlenza, a la Coordinadora de Epidemiología del Ministerio de Salud de la Ciudad Autónoma de Buenos Aires y a Teresa Velich, responsable del Departamento de Vigilancia Alimentaria del Instituto Nacional de Alimentos (INAL) por el diálogo sobre el sistema de salud de la Argentina.

Al Convenio de Cooperación Educativa entre Brasil y Argentina del Programa Binacional de Centros Asociados de Posgrados Brasil/Argentina.

\section{Colaboradores}

Rosana Aparecida Garcia responsabilizou-se por todas as etapas do trabalho. Solange L' Abbate responsabilizou-se pela revisão teórico-metodológica. Jorge Arakaki responsabilizou-se pela supervisão da coleta de dados e discussão dos resultados em campo.

\section{Referências}

1. Lourau R. A Análise Institucional. 2a ed. Petrópolis: Vozes; 1995.

2. Lourau R. O instituinte contra o instituído. In: Altoé $S$, organizadora. René Lourau: analista institucional em tempo integral. São Paulo: Hucitec; 2004. p. 47-65.

3. Lourau R. René Lourau na UERJ. Análise institucional e práticas de pesquisa. Mnemosine. 2007; 3(2):77-92.

4. L'Abbate S. Análise institucional e Saúde Coletiva. Cienc Saude Colet. 2003; 8(1):26574.

5. Belmartino S. Nuevas Reglas de juego para la atención médica en la Argentina ¿Quién será el árbitro? Buenos Aires: Lugar; 1999.

6. Habermas J. Problemas de legitimación en el capitalismo tardío. Buenos Aires: Amorrortu; 1975.

7. Offe C. Contradiction of the Welfare State. Cambridge: IMS Press; 1984.

8. Offe C. Disorganized capitalism. Cambridge: Polity Press; 1985.

9. Alazraqui M, Mota E, Spinelli H. Sistemas de Información en Salud: de sistemas cerrados a la ciudadanía social - un desafío en la reducción de desigualdades en la gestión local. Cad Saude Publica. 2006; 22(12):2693- 702.

10. Spinelli H. El proyecto político y las capacidades de gobierno. Rev Salud Colect. 2012; 8(2):107-30.

11. Baremblitt GF. Compêndio de Análise Institucional e outras correntes: teoria e prática. Rio de Janeiro: Rosa dos Ventos; 1992. 
12. Garcia R. Parcerias entre instituições de ensino e serviços de saúde do Distrito Sudoeste de Campinas: um olhar sobre os (des) encontros [dissertação]. Campinas (SP): Faculdade de Ciências Médicas, Universidade Estadual de Campinas; 2009.

13. Barros RB. Institucionalismo e dispositivo grupal. In: Rodrigues HBC, Altoé S, organizadores. Saúde Loucura, n. 8; análise institucional. São Paulo: Hucitec; 2004. p. 65-78.

14. Lourau R. Implicação e sobreimplicação. In: Altoé S, organizadora. René Lourau: analista institucional em tempo integral. São Paulo: Hucitec; 2004. p. 186-98.

15. Paulon S. A Análise de Implicação como ferramenta na Pesquisa Intervenção. Psicol Soc. $2005 ; 17(3): 18-25$.

16. Pezzato LM. Encontros, instituições e sujeitos em análise: a alta pactuada em saúde bucal [tese]. Campinas (SP): Faculdade de Ciências Médicas, Universidade Estadual de Campinas; 2009.

17. Lourau R. René Lourau na UERJ: análise institucional e práticas de pesquisa. In: Rodrigues HB, organizadores. René Lourau na UERJ. Rio de Janeiro: UERJ; 1993. p. 66-86.

18. Merhy EE, Franco TB. Mapas analíticos: um olhar sobre a organização e seus processos de trabalho. In: Carvalho S, organizador. Conexões. São Paulo: Hucitec; 2009. p. 301-21.

19. Costa EA. Vigilância Sanitária: defesa e proteção da Saúde. Normas de organização e normas de defesa e proteção da saúde [tese]. São Paulo (SP): Faculdade de Saúde Pública, Universidade de São Paulo; 1998. v. 3.

20. Ministerio de Salud. Presidencia de la nación. Manual de normas y procedimientos de Vigilancia y Control de Enfermedades de Notificación Obligatoria de la Nación. Buenos Aires: Ministerio de Salud; 2007.

21. Campos GWS. Saúde Pública e Saúde Coletiva: campo e núcleo de saberes e práticas. Cienc Saude Colet. 2000; 5(2):219-30.

22. Merhy EE, Feuerwerker L, Ceccim, RB. Educación Educación Permanente en Salud: una estrategia para intervenir en la micropolítica del trabajo en salud. Rev Salud Colect. 2006; 2(2):147-60.

23. Yedlin G. La Nación y las provincias formamos un solo equipo. Argent Salud. 2010; $1(6): 6-7$.

24. Matus C. Adiós, señor presidente. Buenos Aires: Universidad Nacional de Lanús; 2007.

Garcia RA, L'Abbate S, Arakaki J. Estratégias de articulação entre Atenção Primária e Vigilância em Saúde e a interface entre os sujeitos. Interface (Botucatu). 2015; 19(54):431-42.

Trata-se de estudo qualitativo com objetivos de descrever as articulações entre a Vigilância em Saúde e a Atenção Primária da Saúde (APS) e os dispositivos utilizados para que estes processos se efetivem. A coleta de dados foi realizada por meio da observação participante do sistema de Vigilância em nível nacional e do município de Moreno província de Buenos Aires. Os dados foram registrados em um diário de campo e os resultados analisados a partir de alguns conceitos elaborados por Lourau, na Análise Institucional, como analisador, instituição e implicação. Os resultados mostraram que a circulação de afetos e de subjetividades entre os sujeitos envolvidos no processo facilita a criação de dispositivos de articulação entre as áreas e jurisdições. A importância deste estudo consiste em um olhar externo que contribui para refletir os processos de trabalho.

Palavras-chave: Saúde Pública. Atenção Primária em Saúde. Atenção em Saúde.

Recebido em 20/03/14. Aprovado em 11/09/14. 